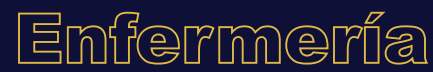

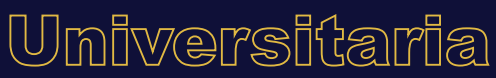

\section{Adaptación cultural y validación de Parental Stressor Scale: Neonatal Intensive Care Unit en padres mexicanos}

\author{
Cultural adaptation and validation of \\ the Parental Stressor Scale: Neonatal \\ Intensive Care Unit in Mexican parents
}

\section{Adaptação cultural e validação de Parental Stressor Scale: Neonatal Intensive Care Unit em pais mexicanos}

\author{
V. Dominguez-Martínez ${ }^{\text {a1* }}$, I. Cortés-Escárcega ${ }^{\text {b2 }}$ \\ ORCID: \\ a $0000-0001-7194-0874$ \\ boooo-0003-2403-9770
}

${ }^{1}$ Servicio Neonatología, Hospital General Dr. Manuel Gea González, Ciudad de México, México

${ }^{2}$ Facultad de Estudios Superiores Zaragoza, Universidad Nacional

Autónoma de México, Ciudad de México, México

Recibido: 22 abril 2019/Aceptado: 12 septiembre 2019

\begin{abstract}
RESUMEN
Introducción: La hospitalización de un recién nacido prematuro en la Unidad de Cuidados Intensivos Neonatales (UCIN), constituye un suceso estresante para los padres, como consecuencia de factores ambientales y psicosociales de la UCIN. Así, la identificación del grado de estrés de los padres a través de instrumentos adaptados y validados a su contexto, establecerá protocolos para disminuirlo durante la hospitalización de su hijo.

Objetivo: Adaptar culturalmente y validar la Escala de Estrés Parental: Unidad de Cuidados Intensivos Neonatales (PSS: NICU) para su aplicación en un grupo de madres y padres mexicanos.
\end{abstract}

\footnotetext{
*Autor para correspondencia. Correo electrónico: vdominguezj12@gmail.com https://doi.org/10.22201/eneo.23958421e.2019.4.641

1665-7063/@ 2019 Universidad Nacional Autónoma de México, Escuela Nacional de Enfermería y Obstetricia. Este es un artículo Open Access bajo la licencia CC BY-NC-ND (http://creativecommons.org/licenses/by-nc-nd/4.0/).
} 
Métodos: Estudio descriptivo y transversal, con una muestra intencional de 130 padres de recién nacidos prematuros hospitalizados en la Unidad de Cuidados Intensivos Neonatales. El estrés parental se midió utilizando la PSS: NICU, que fue adaptada y validada para su aplicación en padres mexicanos. La información se procesó con el paquete estadístico SPSS v.24. Resultados: El instrumento adaptado al contexto cultural en población mexicana presentó una consistencia interna de .945, probando así su confiabilidad para su aplicación en padres mexicanos. El estrés parental total promedio fue de $2.48 \pm .986$, considerado poco estresante. Conclusiones: La adaptación cultural y validación de la PSS: NICU en población mexicana, resultó una herramienta válida y confiable para su aplicación en padres mexicanos. El factor más asociado con el incremento del nivel de estrés fue la alteración del rol parental, por lo que es importante establecer intervenciones dirigidas a mejorar la participación de los padres que apunten hacia el bienestar emocional de los mismos.

Palabras clave: Estrés Psicológico; recién nacido prematuro; padres; unidad de cuidados intensivos neonatales; estudios de validación; México.

\begin{abstract}
Introduction: Considering the related environmental and psychosocial factors, the hospitalization of premature newborns in the Neonatal Intensive Care Unit (NICU) constitutes a stressful event for the parents; because of this, the estimation of the degree of this stress, by means of adapted and validated instruments, can help establishing a more precise protocol approach to address the problem.

Objective: Culturally adapt and validate the Parental Stress Scale: Neonatal Intensive Care Unit (PSS: NICU) for application in a group of Mexican mothers and fathers.

Methods: This is a descriptive and transversal study on a by-intention sample of 130 parents of premature newborns hospitalized in a Neonatal Intensive Care Unit. The associated parent stress was estimated using the PSS: NICU, which was adapted and validated to Mexican parents. Data were processed using SPSS v.24.

Results: The instrument, which was adapted to the mexican population, showed an internal consistency of 0.945 , demonstrating sound reliability. The average total parental stress was $2.48 \pm 0.986$, which was considered as fairly stressful.

Conclusions: The cultural adaptation and validation of the PSS: NICU, to the Mexican population, resulted in a valid and reliable tool which can be used in Mexican parents. The factor with the highest association to the level of stress was Alteration to the Parental Role. Therefore, it is important to establish interventions aimed at improving the emotional status of parents undergoing this kind of stressful events.
\end{abstract}

Keywords: Stress, psychological; infant, premature; parents; intensive care units, neonatal; validation studies; Mexico.

\title{
RESUMO
}

Introdução: A hospitalização de um recém-nascido prematuro na Unidade de Cuidados Intensivos Neonatais (UCIN) constitui um evento estressante para os pais, isto como consequência de fatores ambientais e psicossociais da UCIN. Assim, a identificação do grau de estresse dos pais, através de instrumentos adaptados e validados no seu contexto, estabelecerá protocolos para diminui-lo durante a hospitalização de seu filho.

Objetivo: Adaptar culturalmente e validar a Parental Stressor Scale: Neonatal Intensive Care Unit (PSS:NICU) para sua aplicação em um grupo de mães e pais mexicanos. 
Métodos: Estudo descritivo e transversal, com uma amostra intencional de 130 pais de recém-nascidos prematuros hospitalizados na Unidade de Cuidados Intensivos Neonatais. O estresse parental se mediu utilizando a PSS: NICU, que foi adaptada e validada para sua aplicação em pais mexicanos. A informação processou-se com o pacote estatístico SPSS v.24.

Resultados: O instrumento adaptado no contexto cultural na população mexicana apresentou uma consistência interna de .945, provando assim sua confiabilidade para sua aplicação em pais mexicanos. O estresse parental total médio foi de $2.48 \pm .986$, considerado pouco estressante.

Conclusões: A adaptação cultural e validação da PSS: NICU, em população mexicana, resultou uma ferramenta válida e confiável para sua aplicação em pais mexicanos. O fator mais associado com o incremento do nível de estresse foi a alteração do rolo parental, pelo que é importante estabelecer intervenções dirigidas a melhorar a participação dos pais que apontem ao bem-estar emocional deles.

Palavras chave: Estresse Psicológico; recém-nascido prematuro; pais; unidade de cuidados intensivos neonatais; estudos de validação; México.

\section{INTRODUCCIÓN}

Según estimaciones de la OMS cada año nacen 15 millones de niños antes de llegar a término¹. En México, la DGIS (Dirección General de Información en Salud) en el año 2017 reportó 2,064,507 nacimientos, de los cuales 148,986 fueron prematuros ${ }^{2}$. A nivel mundial la prematurez es la primera causa de mortalidad en niños menores de cinco años y cada día las tasas de nacimientos prematuros están en aumentoํ. Por tanto, ha provocado que éstos requieran atención especializada de las diferentes instancias de salud y que sean sometidos a una serie de medidas terapéuticas que resultan agresivas para el recién nacido, tales como la ventilación mecánica, los accesos vasculares, así como una serie de dispositivos altamente tecnificados que se hacen presentes en la atención y su cuidado.

Dentro de este marco, el entorno se vuelve hostil para el recién nacido y sus padres; pues por un lado, tenemos su ingreso a una unidad con factores estresantes (ruido, luz o manipulaciones excesivas) y por otro, la percepción de los padres al verlo en un cuna rodeado de equipos altamente tecnificados y bajo un pronóstico incierto, lo que ocasiona temor e incertidumbre al dejar a su hijo al cuidado de otras personas 3 .

Si bien los avances en el cuidado del neonato han permitido la supervivencia de recién nacidos cada vez más prematuros, también ha condicionado un incremento en los ingresos a las Unidades de Cuidados Intensivos Neonatales, situación que genera un impacto emocional en los padres, tanto por el estado de salud de su recién nacido4, como por las características ambientales de la unidad5, lo que provoca en ellos altos niveles de estrés, ante los cuales no se encuentran preparados.

La hospitalización de un recién nacido en la Unidad de Cuidados Intensivos Neonatales (UCIN) constituye así un suceso estresante para los padres. De acuerdo con la literatura, éstos presentan síntomas de estrés derivados de factores como: la condición de salud y vulnerabilidad del recién nacido, las características y el ambiente de la unidad en donde se encuentran hospitalizados y la separación del binomio padres-hijo ${ }^{6,7}$. Lo anterior se potencia aún más sí su hijo es prematuro, ya que éstos tienen un mayor riesgo de resultados adversos posteriores en diversos aspectos del desarrollo8.

Por otra parte, el estrés conceptualizado como una respuesta general del organismo ante demandas internas o externas que en un principio resultan amenazantes, consiste básicamente en una 
movilización de recursos fisiológicos y psicológicos para poder afrontar tales demandas ${ }^{9}$ y puede generar en los padres reacciones diversas que interfieran en la interacción entre ellos, su hijo y su familia ${ }^{10}$.

Además, estudios han determinado que la hospitalización de un hijo impacta en la dinámica y en la estructura familiar y puede causar inseguridad, frustración, estrés, miedo, incertidumbre y culpa, especialmente en los padres ${ }^{11}$, así como sentimientos de exclusión en el cuidado de su recién nacido ${ }^{12,13}$.

En este sentido, la participación de la enfermera debe estar orientada a identificar manifestaciones de estrés en los padres y promover intervenciones que favorezcan resultados psicosociales positivos, con el fin de disminuir los sentimientos de estrés, ansiedad y pérdida de control. De ahí que las intervenciones deben centrarse en la familia y la atención de apoyo en el desarrollo ${ }^{14}$.

La determinación del grado de estrés de padres de recién nacidos relacionada con el entorno de la UCIN, se ha evaluado a través de la Parental Stress Scale: Neonatal Intensive Care Unit (PSS: NICU), que ha sido traducida y validada en otros países y bajo otro contexto cultural ${ }^{15-18}$. No obstante; ninguna versión en población mexicana está disponible, por lo que es fundamental para las enfermeras contar con una herramienta objetiva, con constructos claramente definidos, que permitan evaluar con mayor precisión la percepción de los padres ante los elementos estresores presentes en el entorno de la Unidad de Cuidados Intensivos durante la hospitalización de su recién nacido, en coherencia con su contexto sociocultural y que sirva como medio para orientar acciones de atención a la salud dentro del medio hospitalario y fomentar la participación de los padres en el cuidado del recién nacido durante su estancia en la Unidad.

Bajo este contexto, se tuvo como objetivo adaptar culturalmente y validar la escala de estrés parental en una Unidad de Cuidados Intensivos Neonatales con un grupo de madres y padres mexicanos en un hospital del sector público, que permitió la cuantificación del grado de estrés en población mexicana, así como la identificación de fuentes de estrés de los padres relacionados con el entorno de la UCIN, esencial para proponer estrategias eficaces de promoción de un cuidado óptimo.

\section{MÉTODOS}

Estudio descriptivo y transversal ${ }^{19}$. La población de referencia fueron madres y padres de recién nacidos prematuros hospitalizados en la Unidad de Cuidados Intensivos Neonatales del Hospital General Dr. Manuel Gea González de la Ciudad de México, en el periodo comprendido de septiembre de 2017 a octubre de 2018, con un muestreo intencional o de Juicio²0.

El instrumento utilizado fue la Parental Stressor Scale: Neonatal Intensive Care Unit (Escala de Estrés Parental: UCIN), que fue desarrollada por Miles, Funk y Carlson (1993) ${ }^{21}$, para medir el grado de estrés de los padres ante factores estresantes durante la hospitalización de su hijo recién nacido; la cual consta de 26 ítems distribuidos en tres subescalas:

Imágenes y sonidos (conformada de 5 ítems); ésta evalúa el estrés percibido por los padres ante el ambiente físico de la unidad.

Apariencia, tratamiento y comportamiento del recién nacido (consta de 14 ítems); que evalúan la forma en cómo se comporta el recién nacido con relación al tratamiento.

Rol parental (conformada por 7 ítems); evalúa la percepción de las madres y padres ante la pérdida del vínculo con su recién nacido.

Los padres son interrogados para evaluar los reactivos, en cada una de las subescalas éstos tienen que indicar en cinco puntos, a través de una escala tipo Likert, sus experiencias estresantes en relación 
con los elementos de cada subescala; donde la puntuación 1 se refiere a no estresante, 2 poco estresante, 3 moderadamente estresante, 4 muy estresante y 5 extremadamente estresante. Cabe señalar que esta escala ha sido traducida y validada en otros países y bajo otros contextos culturales ${ }^{10,15-18}$.

\section{Validez de contenido}

Para la adecuación y validación de contenido ${ }^{22}$ del instrumento se llevó a cabo la traducción de la Parental Stressor Scale: Neonatal Intensive Care Unit (PSS: NICU) del idioma original (inglés) al español, por un traductor certificado y cuya lengua materna es el español. La validez de expertos se realizó por un grupo de profesionales de Enfermería, expertas clínicas y especializadas en Enfermería del neonato de dos instituciones del sector público, quienes brindan atención directa a recién nacidos prematuros y mantienen contacto directo con los padres de éstos, además de una psicóloga y colaboradora directa con los padres de los recién nacidos hospitalizados.

En el instrumento se evaluó comprensión, claridad, precisión y pertinencia. Cada juez tenía la libertad de realizar adecuaciones a la redacción de los ítems que consideraba confusos para conservar la congruencia con el factor evaluado.

Se adecuaron los ítems 7 y 17 de la dimensión de apariencia, comportamiento y tratamiento del bebé y con base en las observaciones realizadas por el panel de expertos, se obtuvo la primera versión del instrumento y se aplicó una prueba piloto a un grupo de madres y padres mexicanos de recién nacidos prematuros que cubrían las mismas características de la población en estudio. Se determinó la factibilidad de los procedimientos, condiciones de aplicación y claridad de los ítems para eliminar de la prueba final posibles variables confusoras y así determinar su aplicación en el escenario de estudio, obteniendo la versión final del instrumento. Así mismo, se realizó la retrotraducción para determinar diferencias semánticas y conceptuales importantes entre la escala original y la versión obtenida.

\section{Validez de Constructo}

Para la validez del instrumento se realizó un análisis factorial exploratorio utilizando el método de extracción de análisis de componentes principales con rotación varimax.

Para establecer la confiabilidad se utilizó el Coeficiente Alfa de Cronbach. Se consideró para su interpretación valores por arriba de 0.70 , aceptable para propósitos exploratorios ${ }^{19}$.

La muestra estuvo conformada por 130 madres y padres de 87 recién nacidos ingresados en la UCIN. Se incluyeron madres y padres (de recién nacidos menores a 36 semanas de gestación ingresados a la Unidad de Cuidados Intensivos Neonatales que cursaran entre el tercero y quinto día de vida, con o sin apoyo ventilatorio o medidas invasivas adicionales) sin distinción de edad y quienes, al ser interrogados, refirieran ser nacidos en México y tener más de 15 años de viviendo en el país, que supieran leer y escribir, así como que aceptaran participar en el estudio. Se excluyeron a las madres y padres de recién nacidos prematuros con cuidados paliativos, ya que en ellos se presentan reacciones aflictivas típicas ${ }^{23}$ (culpa, pena intensa y perdida de esperanzas) que pueden sesgar los resultados debido a la formación del duelo anticipado.

Ambos padres fueron entrevistados de manera separada para que no interviniera la percepción del otro en sus respuestas. La aplicación del instrumento se realizó treinta minutos previos a la hora de visita y del informe médico, en un área fuera de la Unidad de Cuidados Intensivos Neonatales para capturar la sensación de estrés antes de tener información relacionada con el estado de salud de su recién nacido. 
Para el análisis de resultados se utilizó estadística descriptiva y se procesaron a través del paquete estadístico SPSS Versión 24.

\section{RESULTADOS}

La edad promedio de las madres y padres de los recién nacidos prematuros hospitalizados fue de 29 \pm 7.8 , con una edad mínima de 15 y máxima de 53 años. Los participantes reportaron ser empleados en un $28 \%$, amas de casa $58 \%$, comerciantes $15 \%$, desempleados $7 \%$, profesionistas $2 \%$ y estudiantes $3 \%$.

Tabla 1. Distribución de variables sociodemográficas de la población estudiada

\begin{tabular}{llcc}
\hline Variable & Categoría & Frecuencia & Porcentaje \\
\hline Parentesco & Madres & 70 & 54 \\
\hline & Padres & 60 & 46 \\
\hline Edad & 15 a 20 años & 23 & 18 \\
\hline & 21 a 25 años & 23 & 18 \\
\hline & 26 a 30 años & 27 & 21 \\
\hline & 31 a 35 años & 29 & 22 \\
\hline & Más de años 40 años & 19 & 14 \\
\hline Estado Civil & Soltero & 9 & 7 \\
\hline & Casado & 10 & 8 \\
\hline Escolaridad & Unión libre & 39 & 30 \\
\hline & Primaria & 81 & 62 \\
\hline & Secundaria & 21 & 16 \\
\hline & Bachillerato & 54 & 36 \\
\hline & Licenciatura & 47 & 5 \\
\hline & Posgrado & 6 & 1 \\
\hline
\end{tabular}

Con relación a las variables clínicas de los recién nacidos, los rangos de edad gestacional que predominaron fueron de 33 a 36 semanas de gestación con un $65 \%$ y una edad gestacional mínima de 26 semanas y una máxima de 36 semanas, mientras que los rangos por peso se expresaron de la siguiente manera: 400-1000 gramos 12\%, 1001-1500 gramos $24 \%$, 1500-2000 gramos 32\%, 2001-2500 gramos $29 \%$ y más de 2501 gramos $3 \%$.

Por su parte, el análisis de constructo reveló cinco factores con una varianza de 68.7\%. (Tabla 2) El instrumento utilizado y adaptado al contexto cultural de la población mexicana presentó un nivel de consistencia interna de 0.945 , probando así su confiabilidad para su aplicación en padres y madres mexicanos.

Tabla 2. Confiabilidad del Instrumento por Dimensiones

\begin{tabular}{lll}
\hline Dimensión & Alfa de Cronbach & \\
\hline & Adaptación* & Escala original** \\
\hline Imágenes y sonidos & .82 & .73 \\
\hline $\begin{array}{l}\text { Apariencia, tratamiento y } \\
\text { comportamiento del recién nacido }\end{array}$ & .93 & .83 \\
\hline Rol parental & .90 & .83 \\
\hline
\end{tabular}

Fuente: ${ }^{* *}$ Miles M, Funk S, Carlson J. Parental Stressor Scale: Neonatal Intensive Care Unit. Nurs Res. 1993;42(3):148-152. *Adaptación: V. Domínguez \& I. Cortés 2017. 
En cuanto a los resultados derivados de la aplicación de instrumento validado, el estrés parental total promedio fue de $2.48 \pm .986$, considerado como poco estresante. El factor más relevante fue la alteración del rol parental. (Gráfica 1).

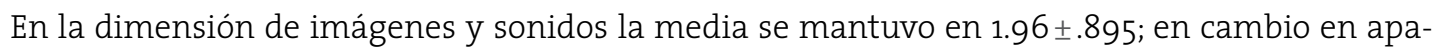
riencia, tratamiento y comportamiento del recién nacido el promedio fue de $2.29 \pm 1.165$ y en el rol parental la media fue de $3.24 \pm 1.242$.

Grafica 1. Nivel de estrés global en madres y padres de recién nacidos prematuros hospitalizados. $\mathrm{N}=130$

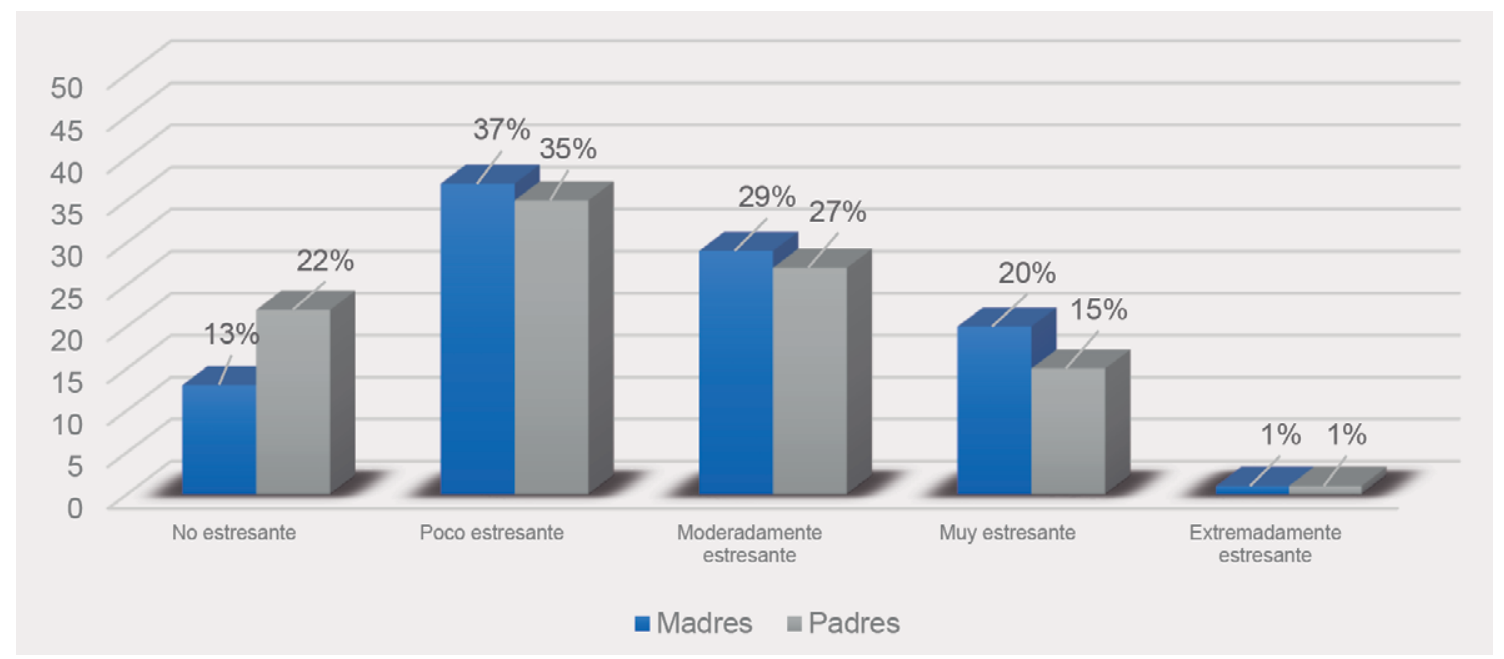

Fuente: Miles M, Funk S, Carlson J. Parental Stressor Scale: Neonatal Intensive Care Unit. Nurs Res. 1993;42(3):148-152. *Adaptación: V. Domínguez \& I. Cortés 2017.

\section{DISCUSIÓN}

Los avances en el cuidado del prematuro han permitido la supervivencia de recién nacidos cada vez más pequeños, que habitualmente se consideraban no viables. Sin embargo; esto se ha convertido en una fuente generadora de estrés para los padres, para quienes el nacimiento prematuro y la hospitalización es un evento inesperado, sobre todo, la separación con sus bebés y la consecuente alteración de su rol de padres ${ }^{24}$.

La identificación de fuentes de estrés a través de escalas validadas al contexto de la población en estudio, ha permitido analizar de manera más objetiva el grado de estrés que perciben los padres ante la hospitalización de su recién nacido, situación que ha sido evaluada dentro de diferentes ámbitos y contextos ${ }^{25}$.

La validación de la escala PSS: NICU para su utilización en madres y padres mexicanos, reportó hallazgos similares a los estudios de aplicación de ésta en su idioma original ${ }^{21}$. Dentro de las subescalas de imágenes y sonidos, apariencia, tratamiento y comportamiento del recién nacido, así como del rol parental, se obtuvo una consistencia interna a través de un alfa de Cronbach de .82, .93 y .90 respectivamente, considerada como buena ${ }^{19}$, es decir, confiablidad adecuada, semejantes con los estudios realizados en Estados Unidos ${ }^{21}$, Argentina ${ }^{10}$ Australia ${ }^{16}$ y Brasil ${ }^{17}$.

Dado que el objetivo de este estudio es la adaptación, validez de contenido y confiabilidad de la versión de la Escala de Estrés Parental: Unidad de Cuidados Intensivos Neonatales para su aplicación en padres mexicanos, obtuvimos información significativa sobre cómo los padres experimentan la 
hospitalización y el nivel de estrés que se genera desde varios aspectos, tanto psicosociales como del entorno, tal como lo identificaron Gonya y colaboradores ${ }^{26}$ en su estudio en donde el estrés materno se ve afectado, por un lado, por la estructura de la unidad y por el otro, afecta la estructura de la enfermería individual en cuanto al cuidado. En consecuencia, resulta imprescindible modificar el actuar del profesional de Enfermería a través de intervenciones dirigidas a la identificación del grado de estrés de los padres y de la forma en cómo viven la hospitalización, ya que esto facilitaría la disminución en los niveles de estrés ante dicha situación ${ }^{27}$.

El ingreso de un recién nacido a la UCIN ha sido identificado por diversos estudios, como un evento estresante para los padres, quienes refieren que existe una pérdida de control sobre su bebé, pues se ven inmersos en factores estresantes derivados del ambiente de la UCIN, del estado de salud de su hijo y de la separación que existe al momento de la hospitalización ${ }^{28,29}$.

Conforme a la teoría de estrés de Magnusen, los factores estresantes definidos como elementos físicos y sociales de una situación que impone demandas y que dan lugar a reacciones como angustia, preocupación y tensión, son percibidos, interpretados y representados cognitivamente por los padres, de acuerdo con las características personales y al medio en el que se desarrollan ${ }^{30}$. Por tanto, considerar escalas validadas permitirá determinar con mayor exactitud la percepción de los padres ante la experiencia estresante de tener un hijo recién nacido hospitalizado.

Con respecto a este estudio, se encontró que el nivel de estrés global percibido por los padres fue poco estresante, en contraste con Carruso ${ }^{10} \mathrm{y}$ Sweet ${ }^{16}$, quienes observaron que los padres perciben la hospitalización de un hijo como moderadamente estresante.

El análisis efectuado para cada subescala arrojó los mismos resultados, tanto en la subescala de imágenes y sonidos, es decir, lo que ven y escuchan dentro de la unidad y la forma en cómo se comporta el recién nacido, situaciones que se perciben como poco estresantes. Por el contrario, en la dimensión relacionada a la alteración del rol de padres, los niveles de estrés se intensificaron, considerándolo muy estresante. Lo cual se refleja en que el promedio más alto lo presentó la alteración del rol parental y dentro de esta dimensión: el estar separado de su bebé, el no poder alimentarlo, sentir impotencia y no ser capaz de abrazarlo.

Turner ${ }^{31}$, Wormald ${ }^{32}$ y Sikorova ${ }^{33}$ reportaron resultados similares, donde observaron que el puntaje promedio más alto fue la alteración del rol de padres y mostraron que una fuente importante de estrés es no poder satisfacer las necesidades básicas de su bebé. Asimismo, estudios cualitativos ${ }^{13,34}$ muestran que los padres perciben que la internación de un hijo los excluye del cuidado y que la falta de interacción genera sentimientos de no pertenencia, teniendo efectos negativos en el vínculo madre-padre-hijo. Esto último se relaciona a las expectativas que los padres se generan antes del nacimiento de su hijo, pues la hospitalización de éste produce alteraciones tanto de los padres como de la unidad familiar, provocando una atmósfera de incertidumbre sobre lo que pasará con el recién nacido y el dejarlo al cuidado de otros.

Teniendo en cuenta lo anterior, la reducción del estrés de las madres y padres debe ser un objetivo fundamental dentro de las Unidades de Cuidados Intensivos, ya que en este aspecto las enfermeras pueden proporcionar información, orientación y apoyo profesional tanto a las madres como a los padres, durante el período de hospitalización de su recién nacido prematuro35. Estudios de intervención han demostrado la efectividad de éstas sobre la disminución del estrés de los padres, algunas se han centrado en el estrés materno y algunas otras hacia el paterno, ambas dirigidas a desarrollar seguridad en el cuidado de su hijo y en su papel de padres 5,36,37. 


\section{CONCLUSIONES}

La falta de equivalencia de instrumentos de medición limita las posibilidades de comparación de una situación específica entre poblaciones de diferentes idiomas y culturas, por lo que la adaptación cultural y validación de instrumentos permite obtener una herramienta de medición equivalente a su versión original, evitando interpretaciones erróneas debido a diferencias culturales y de lenguaje.

La adaptación cultural y validación de la Escala Parental Stress Scale: Neonatal Intensive Care Unit (PSS: NICU) en padres mexicanos, presentó un nivel alto de comprensión en madres y padres con hijos prematuros hospitalizados en la Unidad de Cuidados Intensivos Neonatales. La exclusión de conceptos que resultaban ambiguos, los cambios conceptuales, así como la semántica utilizada, permitieron un texto claramente descrito y comprensible para la totalidad de los padres, quienes a través del análisis descriptivo se encontraron dentro de un nivel educativo medio correspondiente a la población que asiste a instituciones de salud, como la descrita en este estudio, por lo que resultó una herramienta válida y confiable en la práctica clínica, bajo el contexto de madres y padres mexicanos.

La aplicación del instrumento a una población con características similares determinó la factibilidad y claridad de los constructos, así como su aplicación en el escenario de estudio, lo que permitió evaluar con mayor precisión las experiencias estresantes de los padres ante la hospitalización de su hijo prematuro, derivadas tanto del entorno de la UCIN como de las condiciones de salud de su recién nacido y de la separación de éste.

De igual forma, el estudio permitió la identificación de factores asociados con el incremento de los niveles de estrés, derivados del ambiente físico y psicosocial de la UCIN. Uno de los más significativos fue la alteración del rol parental, por lo que es importante establecer intervenciones dirigidas a mejorar la participación de los padres que apunten a mejorar el bienestar emocional de los mismos.

\section{RESPONSABILIDADES ÉTICAS}

A cada uno de los sujetos de estudio se les explicó el propósito y el alcance de la investigación en forma verbal. Se solicitó su consentimiento por escrito para participar en el estudio con apego a las disposiciones éticas de la Ley General de Salud. Titulo $5^{\circ}$. Investigación para la Salud ${ }^{38}$, del Reglamento de la Ley General de Salud en Materia de Investigación para la Salud, Capítulo 3. De la Investigación en Menores edad o incapaces ${ }^{39}$, así como el apego a la doctrina del menor maduro ${ }^{40-42}$, para los casos de padres menores de edad. El estudio fue sometido para su aprobación por los Comités de Investigación y Ética de la institución, con número de registro 41-71-2017.

Conflicto de intereses. Los autores declaran no tener conflictos de intereses.

Financiamiento. No se recibió patrocinio para realizar este estudio.

\section{REFERENCIAS}

1. Organización Mundial de la Salud. Nacimientos prematuros; Ginebra: OMS; 2018. http://bit.ly/2mkQoGK

2. Dirección General de Información en Salud. México: Nacimientos cubos dinámicos; México: DGIS. 2018 http://bit.ly/2nDV6Pb

3. Castro F, Johanson L, Leite de Souza R, Moreira M, Da Conceição E. El primer encuentro del padre con el bebé prematuro en la Unidad de Cuidados Intensivos Neonatales. Index Enferm. 2015; 24(1-2). http://dx.doi.org/10.4321/S1132-12962015000100007 
4. Sola A. Cuidados neonatales: Descubriendo la vida de un recién nacido enfermo. Buenos Aires: Ediciones Médicas; 2011.

5. Turan T, Basbakkal Z, Ozbek S. Effect of nursing interventions on stressors of parents of premature infants in neonatal intensive care unit. J Clin Nurs. 2008; 17(21):2856-66.

https://doi.org/10.1111/j.1365-2702.2008.02307.x

6. Parra Falcón FM, Moncada Z, Oviedo-Soto S, Marquina-Volcanes M. Estrés en los padres de los recién nacidos hospitalizados en la Unidad de Alto Riesgo Neonatal. Index Enferm.2009;18(1):1-6. http://bit.ly/31Vd8v9

7. Vasa R, Eldeirawi K, Kuriakose V, Nair J, Newsom C, Bates J. Postpartum Depression in Mothers of Infants in Neonatal Intensive Care Unit: Risk Factors and Management Strategies. Am J Perinatol.2014;31(5):425-34. https://doi.org/10.1055/s-0033-1352482

8. Soleimani F, Zaheri F, Abdi F. Long-Term Neurodevelopmental Outcomes After Preterm Birth. Iran Red Crescent Med J. 2014; 16(6): e17965. https://doi.org/10.5812/ircmj.17965

9. Buceta J. Bueno A. Estrés, rendimiento y salud. En: Mas-García B.Intervención Psicológica y Salud. Control de estrés y conductas de riesgo. Madrid: Dykinson; 2001.

10. Caruso A. El estrés en los padres ante el nacimiento y la internación de un bebé nacido prematuro en la unidad de cuidados intensivos neonatales. IV Congreso Internacional de Investigación y de Práctica Profesional en Psicología XIX Jornadas de investigación. VIII Encuentro de investigadores en psicología del MECOSUR: Facultad de Psicología.2012. Buenos Aires. Argentina. http://bit.ly/2VkXkPM

11. Marques-dos Santos L, Lopes-de Oliveira I, Santos-Passos S, Santana-Castelo R, Dantas-da Silva J, Dias-lisboa S. Mudanças familiares decorrentes da hospitalização do prematuro em cuidados intensivos: um estudo com puérperas. Rev. baiana enferm. 2013;27 (3):230-8. http://dx.doi.org/10.18471/rbe.v27i3.8684

12. Obeidat $\mathrm{H}$, Bond $\mathrm{E}$, Callister L. The Parental experience of having an infant in the newborn Intensive Care Unit. J Perinat Educ 2009;18(3):23-9. https://doi.org/10.1624/105812409X461199

13. Gallegos-Martínez J, Reyes-Hernández J, Silvan-Scochi CG. El neonato prematuro hospitalizado: un significado para los padres de su participación en la Unidad Neonatal. Rev. Lat-Am. Enfermagem.2013;21(6):136o-6. https://doi.org/10.159o/0104-1169.2970.2375

14. Busse M, Stromgren K, Thorngate L, Thomas K. Parents' responses to stress in the neonatal intensive care unit. Crit Care Nurse 2013;33 (4):52-6o. https://doi.org/10.4037/ccn2013715

15. Mansson C, Jakobsson U, Lundqvist P. Translation and psychometric evaluation of a Swedish version of the parental stressor scale PSS: NICU. Scand J Caring Sci; 2016; 30(1):193-201. https://doi.org/10.1111/scs.12217

16. Sweet L, Mannix T. Identification of parental stressors in an Australian neonatal intensive care unit. NPCHN. 2012;15(2):8-16.

17. Souza S, Dupas G, Baliero MM. Cultural adaptation and validation for the Portuguese language of the Parental Stress Scale: Neonatal Intensive Care Unit (PSS:NICU). Acta Paul Enferm. 2012;25(2):171-6. http://dx.doi.org/10.1590/S0103-21002012000200003

18. Türkan T, Hatice B, Gülay Ö. Determining the Psychometric Properties of the Turkish Version of the Nurse-Parent Support Tool and the Stress Levels of Parents of Premature Infants Hospitalized in the Neonatal Intensive Care Unit. Clin Nurse Spec. 2016;30(3): E1-E1O https://doi.org/10.1097/NUR.0000000000000204

19. Hernández R, Fernández C, Baptista M. Metodología de la Investigación. $6^{a}$ ed. México: McGraw Hill;2014. 
20. Levin J. Fundamentos de estadística en la investigación. 6a. ed. México: McGraw Hill; 2012.

21. Miles M, Funk S, Carlson J. Parental Stressor Scale: Neonatal Intensive Care Unit. Nurs Res. 1993;42(3):148-152.

22. Carvajal A, Centeno C, Watson R, Martínez M, Sanz A. ¿Cómo validar un instrumento de medida de salud? An Sist. Sanit.Navar. 2011; 4(1):63-72.

23. Montesinos A, Roman A, Muñoz M, Elías L. La asistencia al duelo neonatal: diez años de experiencia en una Unidad de Neonatología. Rev. chil pediatr. 2013; 84(6): 650-8.

http://dx.doi.org/10.4067/S0370-41062013000600008

24. Alkozei A, McMahon E, Lahav A. Stress levels and depressive symptoms in NICU mothers in the early postpartum period. J Matern Fetal Neonatal Med. 2014; 27(17):1738-43.

https://doi.org/10.3109/14767058.2014.942626

25. Franck L, Cox S, Allen A, Winter I. Measuring neonatal intensive care unit-related parental stress. J Adv Nurs. 2005; 49(6): 608-15. https://doi.org/10.1111/j.1365-2648.2004.03336.x

26. Gonya J, Harrison T, Feldman K, Stein M, Chawla N. Nursing networks in the NICU and their association with maternal stress: A pilot study. J Nurs Manag. 2019; 27(2):442-9. https://doi.org/10.1111/jonm.12679

27. Ong SL, Abdullah KL, Danaee M, Soh KL, Soh KG, Lee DSK. The effectiveness of a structured nursing intervention program on maternal stress and ability among mothers of premature infants in a neonatal intensive care unit. J Clin Nurs. 2019; 28(3-4): 641-9. https://doi.org/10.1111/jocn.14659

28. Campo-González A, Amador Morán R, Alonso-Uría R, Ballester-López I. Estrés en madres de recién nacido ingresados en la unidad de cuidados intensivos. Rev Cubana Obstet Ginecol. 2018; 44(2):112. http://bit.ly/zoSUuTr

29. Palma E, Von Wussow F, Morales I, Cifuentes J, Ambiado S. Estrés en padres de recién nacidos hospitalizados en una unidad de paciente crítico neonatal. Rev Chil Pediatr. 2017;88(3):332-9. https://doi.org/10.4067/S0370-41062017000300004

30. Magnusson D. Situational determinants of stress: An interactional perspective. En Goldberger L Brenitz S, editores. Handbook of stress Theoretical and clinical aspect. 1a. ed. New York: Free Press; 1982. p. 231-253.

31. Turner M, Chur-Hansen A, Winefield H, Stanners M. The assessment of parental stress and support in the neonatal intensive care unit using the Parent Stress Scale Neonatal Intensive Care Unit. Women Birth. 2015;28(1): 252-8. https://doi.org/10.1016/j.wombi.2015.04.001

32. Wormald F, Tapia J, Torres G, Canepa P, González A, Rodríguez D, et.al. Estrés en los padres de recién nacidos prematuros de muy bajo peso hospitalizados en unidades de cuidados intensivos neonatales: Estudio Multicéntrico. Arch. Argent. Pediatr. 2015;113(4):303-9. http://bit.ly/2VniZa4

33. Sikirova L, Kucova J. The needs of mothers to newborns hospitalised in intensive care units. Biomed Pap Med Fac Univ Palacky Olomouc Czech Repub. 2012;156(4):330-6.

http://dx.doi.org/10.5507/bp.2011.046

34. Borrero Pachón MP. ¿Qué sentimientos producen los cuidados llevados a cabo por la enfermera en los padres de un recién nacido ingresado en una unidad de cuidados intensivos neonatales? Enferm Clin. 2014;24(4):261-2. https://doi.org/10.1016/j.enfcli.2014.03.009

35. Silnes B, Pettersen S, Vardal M, R $\varnothing$ nnestad A. Parents of preterm evaluation of stressand nursing support. J Neonatal Nurs. 2013; 19 (6): 317-26. https://doi.org/10.1016/j.jnn.2013.01.008 
36. Matricardi S, Agostino R, Fedeli C, Montirosso R. Mothers are not fathers: differences between parents in the reduction of stress levels after a parental intervention in a NICU. Acta Paediatr. 2013;102(1): 8-14.

37. Chourasia N, Surianarayanan P, Bethou A, Bhat V. Stressors of NICU mothers and the effect of counseling-experience from a tertiary care teaching hospital, India. J Matern Fetal Neonatal Med. 2013; 26(6):616-8. https://doi.org/10.3109/14767058.2012.743522

38. Secretaría de Salud Ley General de Salud. México: Secretaría de salud, DOF. 12 diciembre 2007. http://bit.ly/31XdRMt

39. Secretaria de Salud. Reglamento de la Ley General de Salud en Materia de Investigación para la Salud. México: DOF 23 diciembre 1986. http://bit.ly/2IyWauM

40. Jorqui-Azofra M. Régimen jurídico de la autonomía de los menores de edad en el marco de las decisiones sanitarias. Rev. Fac. Derecho Méx.2018; 68(272-1):457-500. http://dx.doi.org/10.22201/fder.24488933e.2018.272-1.67621

41. Santiago-Sáez A, Albarrán ME, Perea B. La doctrina del menor maduro. An Pediatr Contin. 2009;7(3):182-5. https://doi.org/10.1016/S1696-2818(og)71125-4

42. Rivera-Ayala L. La figura legal del menor maduro (mature minor) en materia sanitaria a partir del artículo 12 de la Convención sobre los Derechos del Niño. Derecho en sociedad. 2014;6(1):71-90. 